\title{
Structural organization of cardiolipin-containing vesicles as models of the bacterial cytoplasmic membrane
}

\author{
Alessandra Luchini a, ${ }^{+},{ }^{*}$, Domenico Cavasso ${ }^{\text {b }}$, Aurel Radulescu ${ }^{\text {c }}$, Gerardino D’Errico ${ }^{\mathrm{b}, \mathrm{d}}$, \\ Luigi Paduano b,d and Giuseppe Vitiello d,e,*
}
a Niels Bohr Institute, University of Copenhagen, Universitetsparken 5, 2100 Copenhagen, Denmark.
${ }^{b}$ Department of Chemical Science, University of Naples Federico II, Complesso di Monte Sant'Angelo, via Cinthia 4, 80126 Naples, Italy.
${ }^{c}$ Jülich Centre for Neutron Science, Garching Forschungszentrum, Lichtenbergstrasse 1, D-85747 Garching bei München, Germany.
${ }^{d}$ CSGI, Center for Colloid and Surface Science, via della Lastruccia 3, 50019 Sesto Fiorentino (FI), Italy.
${ }^{e}$ Department of Chemical, Materials and Production Engineering, University of Naples Federico II, Piazzale Tecchio 80, 80125 Naples, Italy.

* correspondence to:

Giuseppe Vitiello,

Department of Chemical; Materials and Production Engineering, University of Naples "Federico II" Piazzale Tecchio 80, 80125 Naples, Italy

giuseppe.vitiello@unina.it

\begin{abstract}
Alessandra Luchini
Niels Bohr Institute, University of Copenhagen,

Universitetsparken 5, 2100 Copenhagen, Denmark.

alessandra.luchini@psi.ch
\end{abstract}

\section{Present Address}

$†$ Paul Scherrer Insitut, Forschungsstrasse 111, 5232 Villigen, Switzerland 


\section{Structure of spin-labelled lipids}

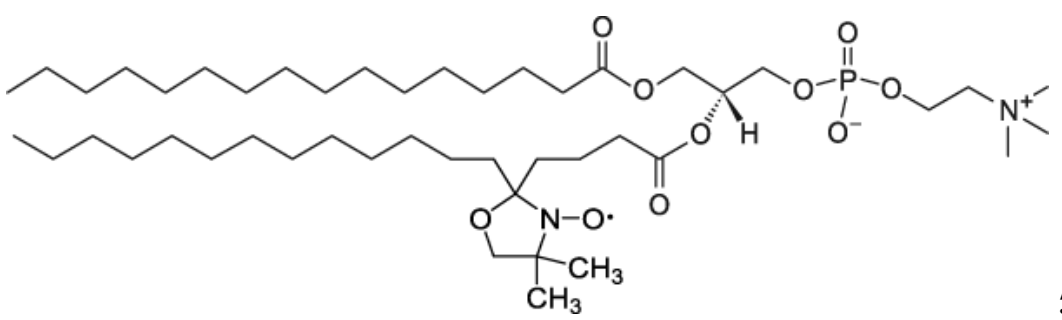

5-PCSL<smiles>CCCCCCCCCCCCCCCCCCC1(CCCCCCCCCCC)OCC(C)(C)N1[O]</smiles>

7-PCSL<smiles>CCCCCCCCCCCCCCCCCC1(CCCCCCCCCC(=O)OC[C@H](O)COP(=O)([O-])OCC[N+](C)(C)C)OCC1(C)C</smiles>

10-PCSL<smiles>CCCCCCCCCCCCCCCCCCCCCC1(CCCCCCCCC(=O)OC[C@H](O)COP(=O)([O-])OCC[N+](C)(C)C)OCC1(C)C</smiles>

14-PCSL

Figure S1 - Molecular structures of $n$-PCSL $(n=5,7,10,14)$ spin-labels used for EPR analysis.

\section{Small Angle Neutron Scattering (SANS) data analysis}

SANS data were analysed with an in-house developed code written in python language. In general, the scattering intensity normalized by the incident beam $(I(q))$ is:

$$
I(q)=\frac{\phi_{p}}{V_{p}} P(q) S(q)+B k g
$$

where $\phi_{p}$ and $V_{p}$ are the particle volume fraction and the particle volume, while $\mathrm{P}(\mathrm{q})$ and $\mathrm{S}(\mathrm{q})$ are the form factor and the structure factor, respectively. Since the vesicle suspensions were sufficiently diluted the structure factor $S(q)=1$ was used.

For the investigated vesicle suspensions, the trend of the experimental data in the low-q region suggested the presence of large vesicle aggregates. Therefore, in the model used to analyse the 
data, we considered the sum of the unilamellar vesicle form factor $\left(P_{\text {vesicle }}\right)$ and a power law, to describe the linear increase of $I(q)$ at low q.

$$
\begin{gathered}
I(q)=\frac{\phi_{\text {shell }}}{V_{\text {shell }}} P_{\text {vesicle }}(q)+A q^{-m}(\mathrm{~S} 2) \\
P_{\text {vesicle }}(q)=\left[3 V_{\text {core }}\left(\rho_{\text {buffer }}-\rho_{\text {shell }}\right) \frac{\sin \left(q r_{\text {core }}\right)-q r_{\text {core }} \cos \left(q r_{\text {core }}\right)}{\left(q r_{\text {core }}\right)^{3}}+3 V_{\text {shell }}\left(\rho_{\text {shell }-} \rho_{\text {buffer }}\right) \frac{\sin \left(q r_{\text {shell }}\right)-q r_{\text {shell }} \cos \left(q r_{\text {shell }}\right)}{\left(q r_{\text {shell }}\right)^{3}}\right]^{2}
\end{gathered}
$$

In equation 2 and 3, $\phi_{\text {shell }}$ and $V_{\text {shell }}$ are the volume fraction and the total volume of the lipids composing the shell of the unilamellar vesicles, respectively. $\rho_{\text {buffer }}$ and $\rho_{\text {shell }}$ are the scattering length density of the buffer, which is composes both the core of the vesicles and the dispersing medium, and of the lipids in the vesicle shell. Finally, $V_{\text {core }}$ is the volume of the vesicle core, and $r_{\text {core }}$ and $r_{\text {shell }}$ are the radius of the vesicle core and the thickness of the vesicle lipid shell, i.e. lipid bilayer thickness. In the power law term of equation $2, A$ is a preexponential factor, and $m$ is the exponent of the power law.

To properly describe the experimental data, equation 2 was modified to take into account both the polydispersity associated to the vesicle size and the instrument resolution. While the lipid bilayer thickness depends only on the lipid molecular structure, the vesicle core radius is expected to have a size distribution, which in the present case was described as Schulz distribution.

$$
\begin{gathered}
I(q)=\frac{\phi_{\text {shell }}}{\bar{V}_{\text {shell }}} \frac{\int P_{\text {vesicle }}(q, r) S(r) d r}{\int S(r) d r} \\
S(r)=\left(\frac{r}{r_{\text {core }}}\right)^{z} e^{-\frac{(z+1) \frac{r}{r_{\text {core }}}}{r_{\text {core }}}}
\end{gathered}
$$

In equations 4 and $5 P_{\text {vesicle }}(q, r)$ is the vesicle form factor calculated in the entire q range for the different radius values, $r$, which are distributed around the mean vesicle radius $\left(r_{\text {core }}\right)$. The parameter $z$ corresponds to $\left(\frac{1}{p^{2}}+1\right)$, where $p$ is the polydispersity. Equation 4 was calculated by considering 50 points in the $\mathrm{r}$ range $\left(r_{\text {core }}-5 p r_{\text {core }}, r_{\text {core }}+5 p r_{\text {core }}\right) \cdot \overline{V_{\text {shell }}}$ is the averaged shell volume calculated by taking into account the Schulz distribution of the vesicle core radius as reported in equation 6 . 


$$
\overline{V_{\text {shell }}}=\frac{\int \frac{4 \pi}{3}\left[\left(r-r_{\text {shell }}\right)^{3}-r_{\text {shell }}{ }^{3}\right] S(r) d r}{\int S(r) d r}
$$

The instrument resolution was approximated by a Gaussian function, as previously reported [Pedersen 1993]. The q-resolution $\left(\sigma=\frac{\Delta q}{q}\right)$ was considered constant in the explored q-range and corresponding to 0.15 . The convolution of the scattered intensity described by equation 4 with the resolution function, considered to be a gaussian, is reported in equation 7 .

$$
\begin{gathered}
I_{\bmod e l}(q)=\frac{\int I(q) G(\langle q\rangle, q) d q}{\int G(\langle q\rangle, q) d q} \\
G(\langle q\rangle, q)=e^{0.5\left(\frac{\langle q\rangle-q}{\sigma q}\right)^{2}}
\end{gathered}
$$

$\langle q\rangle$ is the experimental scattering vector points, while $q$ is the independent variable of the resolution function and 21 points for each $\langle q\rangle$ were used to calculated the integral in equation 7. In the model (equation 7), the scattering length density of the buffer and lipids was calculated according to their chemical composition as $5.9 \cdot 10^{-6} \AA^{-2}$ and $3.2 \cdot 10^{-6} \AA^{-2}$, respectively. Due to the similarity in the chemical composition of the POPE, POPG and CL molecules, the same value for $\rho_{\text {shell }}$ was calculated for the POPE/POPG, POPE/POPG/CL10 and POPE/POPG/CL20 mixtures. The volume fraction of the shell was also initially calculated based on the estimated lipid concentration in the vesicle suspension (i.e. $1 \mathrm{mM}$ ) and subsequently refined to the experimental data. Hence, the remaining model parameters, i.e. $r_{c o r e}, p, r_{\text {shell }}, A, m$, were allowed to vary to produce the best agreement between the model and the experimental data. Errors associated to each of the parameters and reported in Table 1 of the main text were calculated as the square root of the co-variance matrix diagonal multiplied by the reduced $\chi^{2}$.

\section{Computation of the EPR spectra of 14-PCSL}

The spectra of 14-PCSL were analysed by computer simulation using the well-established procedure by Budil et al. [D. E. Budil, S. Lee, S. Saxena and J. H. Freed, J. Magn. Reson., Ser. A, 1996, 120, 155-189], as reported in Figure S2. The main parameters needed in the computation are as follows: (a) the $g_{i i}$ values for the coupling between the electron spin and the magnetic field were those providing the best fitting for all spectra $\left(\mathrm{g}_{\mathrm{ii}}=2.0075,2.006,2.003\right)$; 
(b) the $\mathrm{A}_{\mathrm{ii}}$ main components of the $\mathrm{A}$ tensor for the hyperfine coupling between the unpaired electron spin and the nitrogen nuclear spin were also selected on the basis of the fitting between the experimental and the computed lines. We maintained constant $\mathrm{A}_{\mathrm{xx}}$ and $\mathrm{A}_{\mathrm{yy}}=7.2 \mathrm{G}$, which well fitted all the spectra, while it was needed to change the $A_{z z}$ value. Table $S 1$ shows the $<A>$ $=\left(\mathrm{A}_{\mathrm{xx}}+\mathrm{A}_{\mathrm{yy}}+\mathrm{A}_{\mathrm{zz}}\right) / 3$ values, which give a measure of the environmental polarity at the radical site; (c) the correlation time for the rotational diffusion motion, $\tau$. It was necessary to include in the calculation an anisotropy of motion, also considering a tilt of the rotational axis, but the fitting was good taking constant both the $\tau_{\mathrm{par}}$ value $(13.2 \mathrm{~ns})$, indicative of the steric hindrance of the carbon chin of 14-PCSL, and the tilt angle $\left(70^{\circ}\right)$. Therefore, the main parameter changing from one to another system is the perpendicular value of the correlation time for motion $\left(\tau_{\text {perp }}\right)$, which measures the micro-viscosity at the label environment; and (d) the order parameter, $S$, which measures the order of the lipid bilayer containing 14-PCSL.

From the parameters reported in Table S1, it resulted clear that: (i) the polarity (measured by $<\mathrm{A}>$ ) was quite low, as expected for the lipid zone of the bilayer. The polarity decreased from the absence to the presence of CL and by increasing the CL content; (b) the micro-viscosity (measured by $\tau_{\text {perp }}$ ) was low, as expected for the internal lipid layer. The micro-viscosity decreased from the absence to the presence of CL and by increasing CL content; (c) the lipidbilayer order (measured by $S$ ) was quite high, as expected for a vesicle bilayer. S decreased from the absence to the presence of CL.

\begin{tabular}{c|c|c|c}
\cline { 2 - 4 } & POPE/POPG & $\begin{array}{c}\text { POPE/POPG/ } \\
\text { CL10\% }\end{array}$ & $\begin{array}{c}\text { POPE/POPG/ } \\
\text { CL20\% }\end{array}$ \\
\hline$\tau_{\text {perp }}$ & $0.80 \mathrm{~ns}$ & $0.55 \mathrm{~ns}$ & $0.50 \mathrm{~ns}$ \\
$\tau_{\text {par }}$ & $13.2 \mathrm{~ns}$ & $13.2 \mathrm{~ns}$ & $13.2 \mathrm{~ns}$ \\
$<\mathbf{A}>$ & $14.1 \mathrm{G}$ & $14.0 \mathrm{G}$ & $13.8 \mathrm{G}$ \\
$\mathbf{S}$ & 0.44 & 0.40 & 0.38 \\
\hline
\end{tabular}

Table S1 - Values of the main parameters needed for the simulation of EPR spectra of $n$-PCSL in lipid bilayers of POPE:POPG 70:30 mol/mol in the absence and presence of CL at $10 \mathrm{~mol} \%$ and $20 \mathrm{~mol} \%$. 

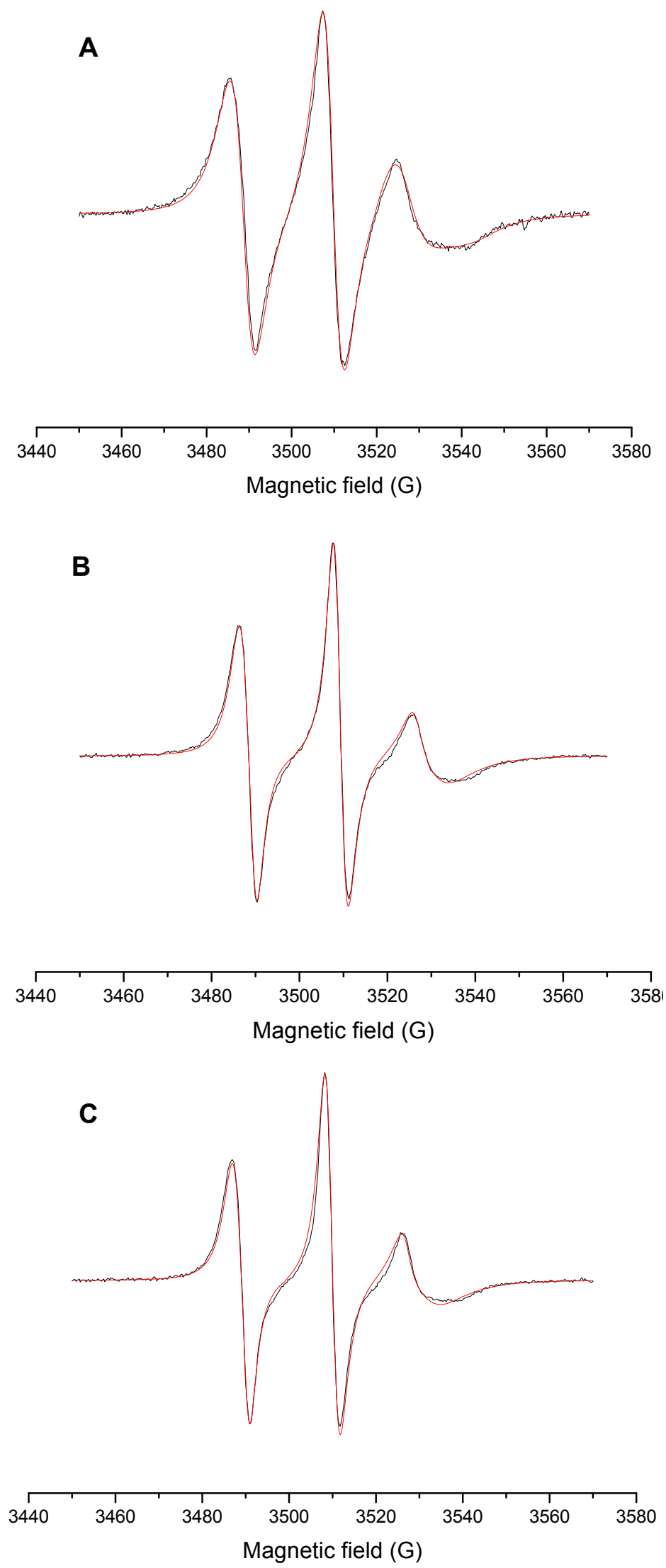

Figure S2 - Experimental (continuous black lines) and simulated (continuous red lines) EPR spectra of 14-PCSL in lipid bilayers of POPE:POPG 70:30 mol/mol in the absence (A) and presence of CL at $10 \mathrm{~mol} \%$ (B) and $20 \mathrm{~mol} \%$ (C). 


\section{EPR characterization of vesicle suspensions in buffer without $\mathrm{Ca}^{2+}$ ions}



Figure S3 - EPR spectra of n-PCSL in lipid bilayers of POPE:POPG 70:30 $\mathrm{mol} / \mathrm{mol}$ in the absence (continuous lines) and presence of CL at $10 \mathrm{~mol} \%$ (pointed lines) and $20 \mathrm{~mol} \%$ (dotted lines), without calcium ions in the buffer solution.

In Figure S3, spectra of n-PCSL inserted in POPE/POPG bilayers without calcium ions are shown. For all lipid systems, 5-PCSL shows a clearly defined axially anisotropic behavior, as detectable by the splitting of the low- and high-field lines, indicating an ordered organization of the outer segments of the lipid acyl chains. As the reporter group is stepped down along the acyl chain (from position 5 to 10), the spectra anisotropy markedly decreases and, in the case of 14-PCSL, a three-line quasi-isotropic spectra is obtained. In order to quantitatively analyze the spectra, the order parameter, $S$, and the isotropic hyperfine coupling constant, ${ }_{\mathrm{N}}^{\prime}$, were evaluated as described in the literature. $S$ is a measure of the local orientational ordering of the labelled acyl chain with respect to the normal to the bilayer surface. ${ }^{a}{ }_{\mathrm{N}}^{\prime}$ is an index of the micropolarity experienced by the nitroxide. Figure $\mathrm{S} 2$ shows the dependence of $S$ on the chain position, $n$, for the $n-P C S L$ spin-labels in bio-membranes of POPE/POPG 70:30 mol/mol in the absence and presence of CL at 10 and 20 mol\%. The corresponding $a_{\mathrm{N}}^{\prime}$ values are reported 
in Table S2.

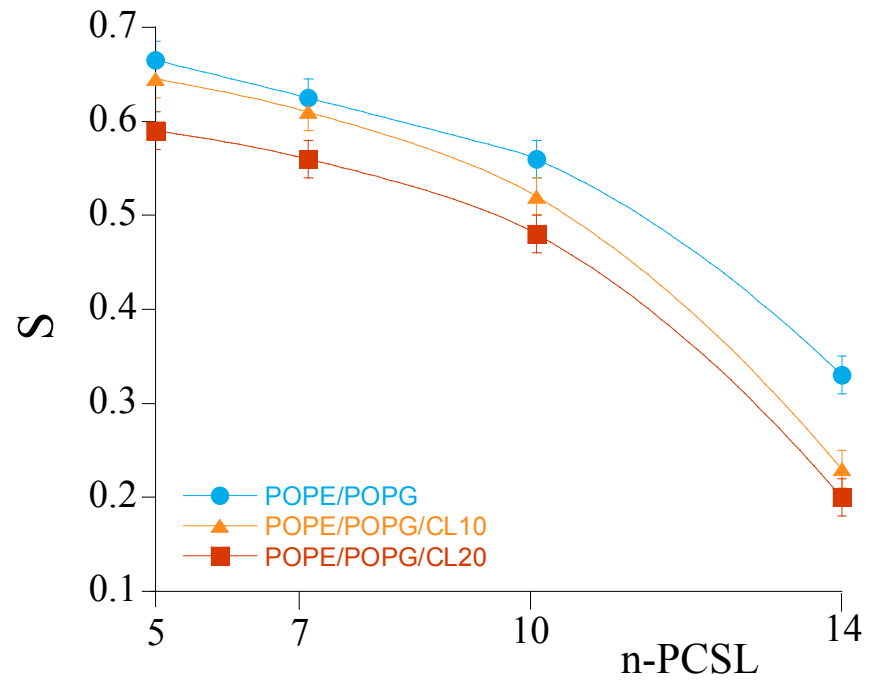

Figure S4 - Profiles of the order parameter, $S$, as function of $n$-position for lipid bilayers of POPE:POPG $70: 30 \mathrm{~mol} / \mathrm{mol}$ in the absence and presence of CL at $10 \mathrm{~mol} \%$ and $20 \mathrm{~mol} \%$, without $\mathrm{Ca}^{2+}$ ions in the buffer solution.

By analyzing the Figure S4, the presence of CL at $10 \mathrm{~mol} \%$ in POPE:POPG bilayers causes a significant increase in the $S$ values of $n$-PCSL spectra. Particularly, the increase in the $S$ values is of the same extent $(\sim 0.5)$ for all spin-label, clearly indicating an increased packing ordering of lipids, involving the whole bilayer. In contrast, the presence of CL at $20 \mathrm{~mol} \%$ in POPE:POPG bilayers causes a different behavior: a decrease of $S$ values was observed for all $n$-PCSL spectra, supporting the fluidizing role of CL at high content. No significant changes were observed in the $a_{N}$ values in the absence and presence of CL in POPE/POPG bilayers.

\begin{tabular}{c|c|c|c}
\cline { 2 - 4 } & POPE/POPG & $\begin{array}{c}\text { POPE/POPG/ } \\
\text { CL10\% }\end{array}$ & $\begin{array}{c}\text { POPE/POPG/ } \\
\text { CL20\% }\end{array}$ \\
\hline 5-PCSL & $0.66 \pm 0.01$ & $0.64 \pm 0.01$ & $0.59 \pm 0.01$ \\
7-PCSL & $0.63 \pm 0.01$ & $0.61 \pm 0.01$ & $0.57 \pm 0.01$ \\
10-PCSL & $0.56 \pm 0.01$ & $0.52 \pm 0.01$ & $0.48 \pm 0.01$ \\
14-PCSL & $0.33 \pm 0.02$ & $0.23 \pm 0.02$ & $0.20 \pm 0.02$ \\
\hline
\end{tabular}

Table S2 - Values of the order parameter, $S$, obtained from experimental EPR spectra of $n$ PCSL in lipid bilayers of POPE:POPG 70:30 mol/mol in the absence and presence of CL at $10 \mathrm{~mol} \%$ and $20 \mathrm{~mol} \%$, without $\mathrm{Ca}^{2+}$ ions in the buffer solution. 